\title{
Boolean Operators to Improve Multi-Objective Evolutionary Algorithms for Designing Optical Networks
}

\author{
Nadja J. da S. Lima, Carmelo J. A. Bastos-Filho, \\ University of Pernambuco, Recife 50720-001 Pernambuco, Brazil \\ Tel: +558131847542e-mail: carmelofilho@ieee.org \\ Danilo R. B. Araújo \\ Federal Rural University of Pernambuco, Recife 52171-900 Pernambuco, Brazil \\ Tel: +558121193842,e-mail: danilo.araujo@ufrpe.br
}

\begin{abstract}
The physical topology design (PTD) of optical networks is frequently accomplished by combining several solutions in an iterative way, especially if meta-heuristics are deployed for this purpose. Suitable operators to recombine information of network topologies aiming at creating innovative options for designing networks are very useful. Operators that preserve desired properties can improve the quality of the meta-heuristics utilized for solving the PTD problem. In this paper, we propose new crossover operators by using the OR and XOR operations to improve multi-objective evolutionary algorithms applied to design optical networks. We performed comparisons between the proposed crossover operators and the traditional uniform crossover. The proposed operators showed to be a suitable alternative to design optical networks. We obtained better solutions or at least solutions with the same quality when compared to solutions achieved by traditional approaches, but the execution time required by our proposal is smaller.
\end{abstract}

Index Terms - Boolean logics, Network design, Optical networks, Genetic Operators, Evolutionary Algorithms, Multi-Objective Optimization.

\section{INTRODUCTION}

The physical topology of a communication network establishes which nodes should be connected through a physical link. These links can be implemented by different technologies, such as copper cables, radio frequency transmission or optical fibers [1]. This problem is often referred as the Physical Topology Design (PTD). The task of finding suitable topologies for real communication networks is not trivial since it involves conflicting optimization objectives, such as to minimize capital cost, to maximize network performance, to increase the network resilience, to reduce the energy consumption, among others [2].

Due to a large number of topology possibilities considering the presence or absence of physical links between each pair of nodes, the PTD problem is classified as a NP-hard problem [3]. To tackle the PTD for real-world networks with a large number of nodes, methods that provide sub-optimal solutions are usually deployed to return satisfactory solutions in a reasonable execution time [4]. Such proposals can be classified into two major groups: heuristics [4], [3] and meta-heuristics [2], [5], [6]. 
A heuristics can be defined as an approach that uses specific information of the problem to obtain a reasonable solution. Most of the works related to heuristics for PTD uses variations of branch exchange (BE) [7] or cut saturation (CS) [8]. Besides, recent studies have also proposed the use of specific geometric graph models that resembles topologies of deployed communication networks [3]. Meta-heuristics are more general algorithms created aiming to solve a particular family of problems. In this case, the algorithm designer only needs to implement small adaptations to apply the metaheuristics to a specific problem. Among many approaches presented in the literature, we can cite two examples of metaheuristics to tackle the PTD problem: simulated annealing [4] and evolutionary algorithms [5], [2]. Some works on metaheuristics are focused in proposing suitable initialization operators [6], [2], but as stated by the authors, there are no noticeable advances related to the proposal of operators to merge existing networks in a smart way to avoid the generation of non-suitable topologies.

Araújo et al. [9] compared five different multi-objective evolutionary algorithms applied to design the physical topology of optical networks. In [9], Araújo et al. used a random generator process to create new network topologies. Araújo et al. [6] also proposed novel operators to improve the initialization process and the mutation over the network topologies. Besides, Araújo et al. [2] presented an improved initialization operator guided by traffic and surrogate models to improve the process of designing optical networks. However, all these previous studies [9], [6], [2] deploy a wellknown and widely used recombination operator named uniform crossover [10]. This recombination operator was not developed a priori to tackle graph-based problems. Thus, it might be the case that this operator is not the most suitable for combining information from different topologies aiming at creating networks that carry topological information from the parent networks. For both heuristics and meta-heuristics, it would be useful to have suitable alternatives to perform this task.

The crossover operator proposed in this work is based on Boolean logics over the adjacency matrix of the physical topology. Previous work was carried out to analyze the advantage of using the AND, OR and XOR operations over adjacency matrix of synthetic networks [11]. In [11], generative procedures of Barabási-Albert (BA), Watts-Strogatz (WS) and Erdos-Renyi (ER) [12] were used to generate synthetic networks with different topological properties to allow the analysis of the impact of the Boolean operators. Lima et.al [11] demonstrated that it is possible to use OR and XOR operations over the adjacency matrix of networks to generate new topologies that preserve sound characteristics of the parent networks. Thus, this work aims to investigate the proposal of new crossover operator based on OR and XOR operations and the use of these crossover operators in a multi-objective evolutionary algorithm deployed to tackle the problem of designing optical networks.

The remainder of this paper is organized as follows. Section II presents a theoretical background on multi-objective evolutionary algorithms. Section III provides a formal description of the problem studied in this article. Section IV provides an explanation regarding the proposed Boolean operators 
for graphs. Section V presents results obtained for the crossover operators concerning hypervolume and execution of simulation time. Finally, Section VI presents the conclusions and future works.

\section{BACKGROUND}

Single objective optimization aims to find values for a vector of decision variables in the space of decision $\Omega$ that minimizes (or maximizes) the value of an independent or objective variable ( $y$ or $f(x)$ ). In this type of problem, the notion of "optimum" is evident since there is just one best value for $y$.

Several real world problems present conflicting objectives. As an example, consider two objective functions in a computer network: minimize costs and minimize the delay to transmit information. One can upgrade the network devices to minimize the delay, but it implies in a higher cost. In this type of problems, one cannot improve one objective avoiding the mitigation of the other. When two or more objective functions are considered, the notion of optimality changes since the goal, in this case, is to find real "trade-offs" rather than a single solution. Multi-objective optimization algorithms aim to find the best Pareto Front for a given problem. The best set of non-dominated solutions is known as Pareto Optimal Set, which is calculated by the Equation (1).

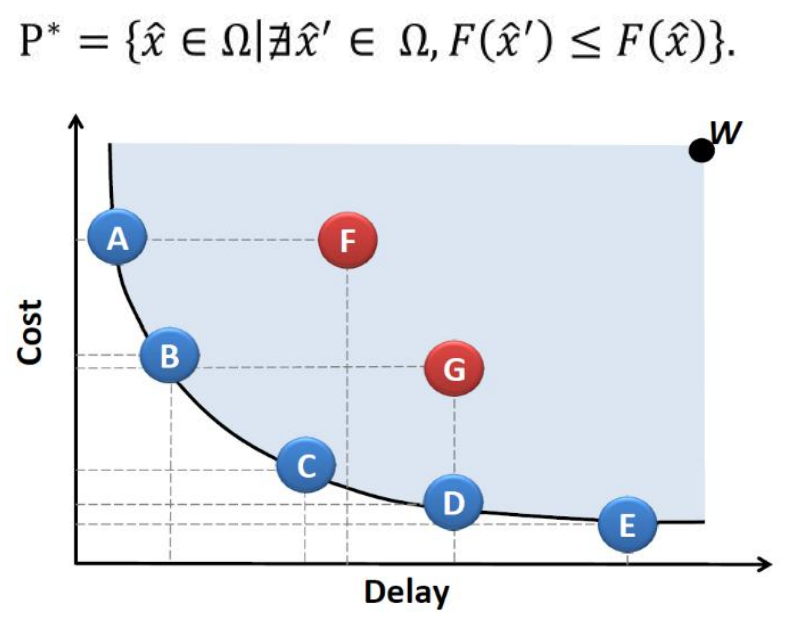

Fig. 1. Example of a Pareto front considering the minimization of two conflicting objective functions.

Fig. 1 illustrates the concept of a tradeoff for a multi-objective problem in a scenario related to the simultaneous minimization of cost and delay for a given telecommunication network. The solution $G$ presents the same delay when it is compared with the solution $D$, but $G$ is more expensive than $D$; the solution $C$ is better than $G$ when both criteria are considered; the solution $C$ is more expensive than solution $D$, but $C$ presents a lower delay value. In fact, the set $P^{*}=\{A, B, C, D, E\}$ is the Pareto optimal set for this example, and this solution set is better than the solutions in the set $P F=\{F, G\}$.

\section{A. Metrics to Assess the Performance of MOEAs}

Several metrics to assess the performance of MOEAs (Multiobjective Evolutionary Algorithms) were already proposed [13], [14]. In this paper, we used MOEAs to solve a real-world problem. Thus, 
we do not have the Pareto optimal set to use as a reference. Some proposed metrics to assess the performance of MOEAs, such as the inverted generational distance (IGD) [14], require the Pareto optimal set to calculate the minimum distance from the obtained solutions to the ones located in the optimal set. Besides, we need to consider metrics to assess both convergence and diversity of solutions. In this paper, we used an indicator that can measure convergence and diversity simultaneously, called hypervolume. The hypervolume (HV) metrics was proposed by Zitzler and Thiele [15] in 1999. It is used to evaluate the area between a reference point $\mathrm{W}$ and the points of the Pareto Front. Better Pareto Fronts with more non-dominated solutions lead to higher hypervolume values. For example, in Fig. 1 the $W$ point is related the worst values for all the objectives and it used as a reference point to calculate the hypervolume. The shadowed area in Fig. 1 is associated to the numerical value of the hypervolume for this scenario.

\section{B. NSGAII Algorithm}

In 2002, Deb et al. [16] proposed the Non-dominated Sorting Genetic Algorithm II (NSGAII). Since 2002, several studies in real-world problems demonstrated that NSGAII is a suitable metaheuristics to solve combinatorial multi-objective optimization problems, especially if two conflicting objectives are considered [17]. To handle problems with more than four conflicting objectives, Deb et al. proposed an improved version of the NSGAII, named NSGAIII [18]. Since the problem studied in this paper is modeled considering two conflicting objectives, we adopted the NSGAII algorithm as the basic metaheuristics to assess the impact our operators. The choice of the NSGAII algorithm was based on previous studies in the design of optical networks. Araújo et al. [9] compared the use of several metaheuristics, including NSGAII, SPEA2, PESAII, MODE, PAES, and showed that the NSGAII and SPEA2 algorithms provide the best results. NSGAII and SPEA2 presented a similar performance for the case of study. Furthermore, other works regarding the design of optical networks applied the algorithm NSGAII and demonstrated that the improvement in the quality of the solutions occurs primarily due to the adjustments related to the genetic operators [6], [2]. Thus, the deployed metaheuristics has a secondary impact on the quality of the obtained Pareto Front.

NSGAII separates the solutions in Pareto Fronts according to the dominance criterion. It uses the crowding distance (CD) method to sort the solutions within the same Pareto Front to preserve the diversity along the Pareto front. The $\mathrm{CD}$ of a solution for two objectives is the size of the square that has as vertices the position of the neighbor solutions in the objective space. If a solution has a small value of $\mathrm{CD}$, this means that this solution is located in a dense region. On the other hand, solutions that have a large $\mathrm{CD}$ value are located in poorly populated regions, and this kind of solution should be preserved. NSGAII ranks the solutions based on the current Pareto Front and crowding distance. Then, the NSGAII algorithm uses elitism at each iteration to maintain the number of individuals constant along the iterations and solely $\mathrm{N}$ individuals are selected for the next iteration of the algorithm. The pseudocode of the NSGAII is shown in Algorithm 1. 
Algorithm 1 - NSGAII Algorithm.

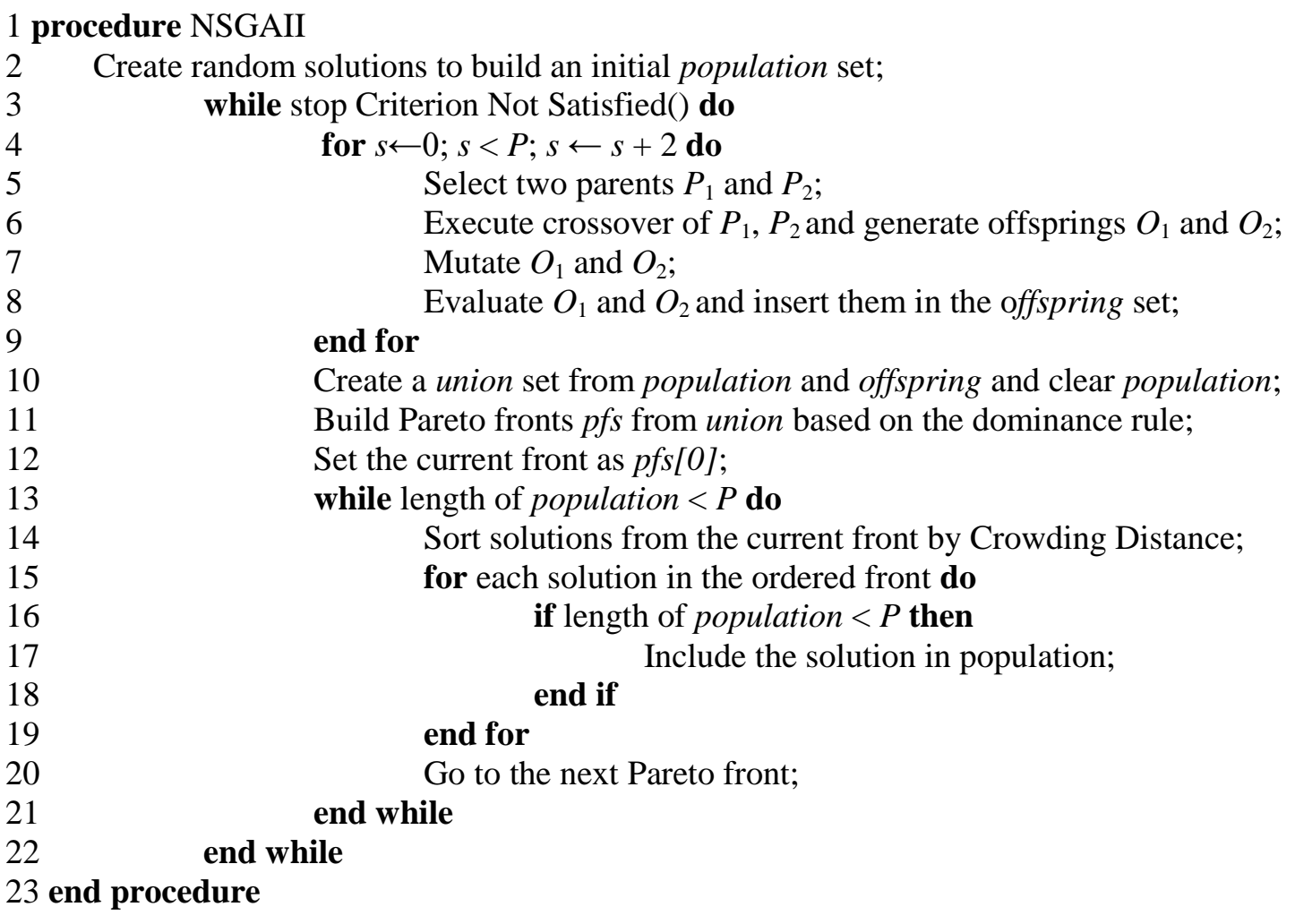

\section{PROBLEM DESCRIPTION}

In this paper we studied the following problem: given the nodes position, the traffic matrix and the Routing and Wavelength Assignment (RWA) algorithm; the goal is to define the physical topology and the specification of the optical devices that should be deployed to simultaneously minimize the CAPEX cost and the overall network blocking probability (BP), constrained by a minimum predefined Quality of Transmission (QoT) required to establish the lightpaths. We chose the following network parameters as decision variables: topology layout (fiber links), the type of Reconfigurable Optical Add-Drop Multiplexers (ROADM) deployed in the network and the number of available wavelength per link. In this work, we used the same cost model of [21]. The cost model considers four different source of cost, as follows: a fixed cost for each wavelength, the fiber deployment cost, the cost of the optical amplifiers and the cost of ROADMs. A generic monetary unit (m.u.) is used to measure the Capital Expenditure (CAPEX) cost.

The positions of the nodes are defined as $P=\left(x_{i}, y_{i}\right)$, in which $(i \in 1,2, \ldots, N), x_{i}$ and $y_{i}$ are Cartesian coordinates of the $i$-th node and $N$ is the total number of nodes. The computational model uses an adjacency matrix $A$, with the elements $a_{\{i, j\}}$, in which $a_{\{i, j\}} \in\{0,1\}$. If $a_{\{i, j\}}=0$, the network nodes $i$ and $j$ are not connected, otherwise they are connected and the correspondent link uses a predefined optical amplifier. We could also have considered the choice of an optical amplifier for each fiber link, but 
Chaves [19] demonstrated that the cost of optical amplifiers is irrelevant to the whole network cost. Thus, we considered a predefined model for the optical amplifiers.

\section{OUR Proposal: A Crossover OPERATOR BASED on BoOleAN LOGICS}

In this paper, we propose the use of Boolean operations deployed as a crossover algorithm. We analyze the results of the network topologies generated by three different Boolean operators: OR, XOR and negative XOR ( XOR).

Let $A$ and $B$ be the adjacency matrix of two networks. These matrices generate a third matrix $C$. Elements $c_{i, j}$ of $C$ are generated by using bitwise operations over the elements $a_{i, j}$ and $b_{i, j}$ from $A$ and $B$, respectively. We analyzed the following operations:

- OR Operation: the element $c_{i, j}$ is set to 1 when the element $a_{i, j}$ or the element $b_{i, j}$ is equal to 1 . $c_{i, j}$ is set to 0 when both elements $a_{i, j}$ and $b_{i, j}$ are equal to 0 .

- XOR Operation: the element $c_{i, j}$ is set to 1 when the elements $a_{i, j}$ and $b_{i, j}$ are different. $c_{i, j}$ is set to 0 otherwise.

- $\sim$ XOR Operation: the element $c_{i, j}$ is set to 1 when the elements $a_{i, j}$ and $b_{i, j}$ are equal. $c_{i, j}$ is set to 0 otherwise.

The proposed crossover operator is shown in Algorithm 2. In Algorithm 2, the $A$ and $B$ arrays contain information regarding the adjacency matrices of the two parent networks. Then, we make the bitwise operations by using the XOR operator to obtain the $C_{l}$ matrix. After this, we perform the bitwise operations using the $\sim$ XOR operator and one obtains the $C_{2}$ matrix. Thus, $C_{1}$ and $C_{2}$ are the offspring generated by combining information from $A$ and $B$.

Other combinations using other cited Boolean operators can be performed only by replacing the XOR and in Algorithm 2. We also tested OR-XOR, OR- XOR and XOR- XOR combinations.

Algorithm 2: crossing procedure using XOR and $\sim \mathrm{XOR}$

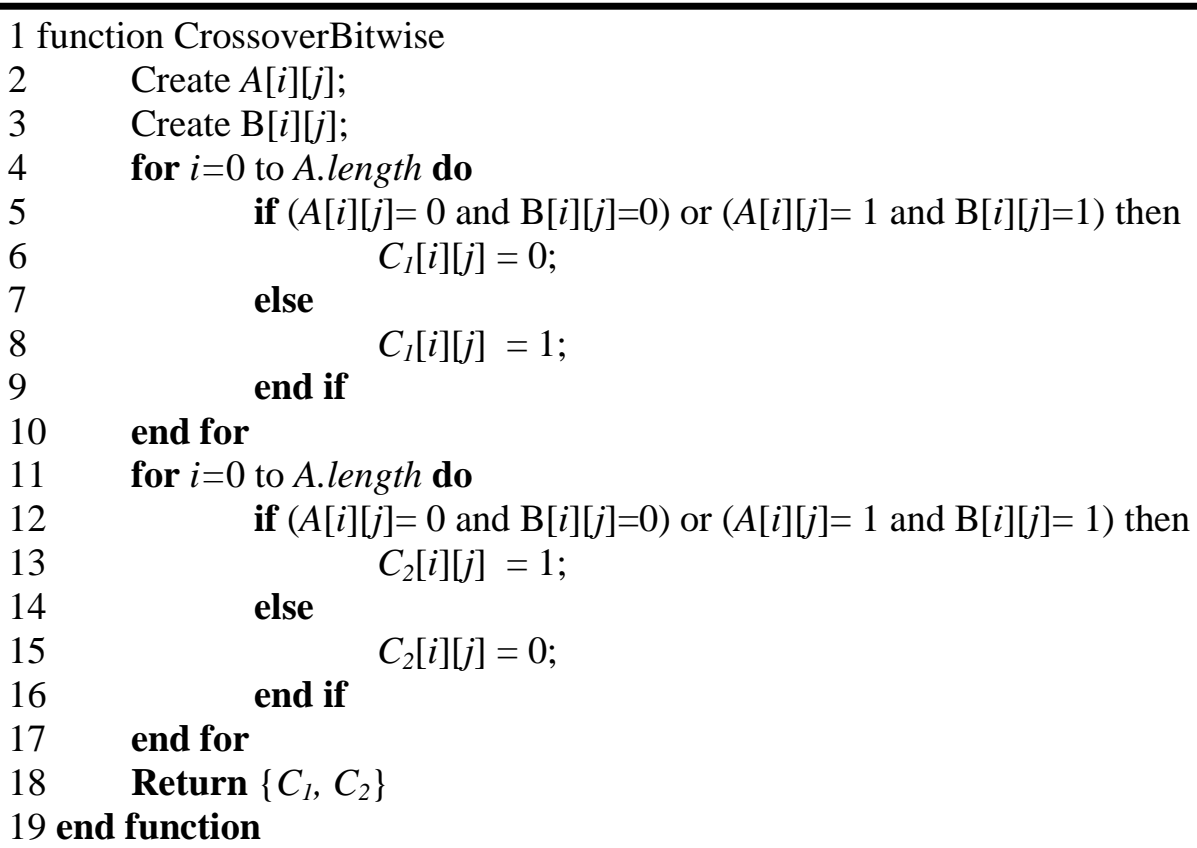

Brazilian Microwave and Optoelectronics Society-SBMO received 08 May 2016; for review 12 May 2016; accepted 06 Sept 2016 Brazilian Society of Electromagnetism-SBMag 
In this paper, our proposal is compared with the uniform crossover [9] applied to a multi-objective evolutionary algorithm, i.e., the NSGAII. The uniform crossover was widely used in previous studies related to the design of optical networks [9], [6], [19], [20], [2].

\section{RESULTS}

In this section, we present the results related to the use of a new crossover operator based on boolean logics when it is deployed in a multi-objective evolutionary algorithm to design optical networks. Our goal is to obtain optical network configurations that present good trade-offs regarding network cost and blocking probability, given as input the geographical locations of the terminal nodes, the traffic demand and the routing and wavelength assignment. We used as decision variables the physical topology (represented by the adjacency matrix), the number of wavelengths and the specification of the ROADMs. We used the same experimental setup deployed in [2]. In this paper, we consider two different geographical coverages, shown in Fig. 2. The first one (Fig. 2a) is related to the design of the well-known 14-node network NSFNet. The second case (Fig. 2b) is related to the design of a 34-node backbone network from Slovenia.

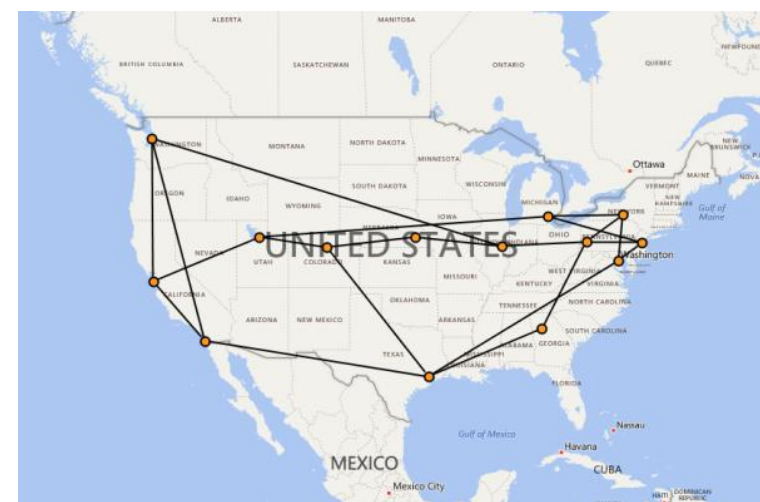

(a)

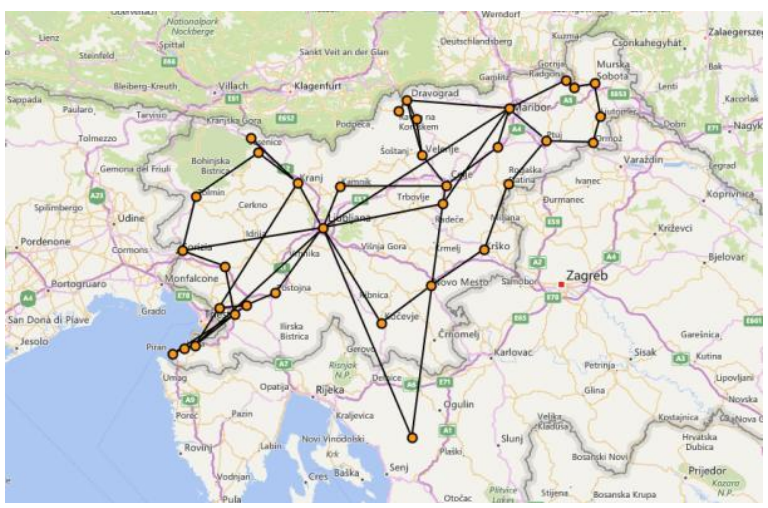

(b)

Fig. 2. Node locations of two different cases used to analyze our proposal.

As mentioned before, our objective space contains two components: cost and network performance. In this paper, we used the SIMTON to estimate blocking probability (BP). An estimate for blocking probability takes in account the Quality of Transmission (QoT) of each lightpath. Besides, the QoT of each lightpath is evaluated by the SIMTON by considering the following physical layer effects: amplified spontaneous emission (ASE) of the amplifiers, gain saturation effect of the amplifiers, ASE saturation in EDFAs, residual chromatic dispersion and ROADM crosstalk. The network parameters used in the simulations of the optical networks are listed in Table I. 
Table I. Network Simulation Parameters.

\begin{tabular}{|c|c|c|}
\hline Parameter & Value & Definition \\
\hline$O S N R_{\text {in }}$ & $40 \mathrm{~dB}$ & Input Optical Signal-to-Noise Ratio \\
\hline$O S N R_{T H}$ & $17 \mathrm{~dB}$ & Minimum OSNR at the receiver \\
\hline$B_{o}$ & $100 \mathrm{GHz}$ & Optical filter bandwidth \\
\hline$\Delta \mathrm{f}$ & $100 \mathrm{GHz}$ & Channel spacing \\
\hline$\lambda_{i}$ & $1529 \mathrm{~nm}$ & First wavelength of the grid \\
\hline$A$ & $0.2 \mathrm{~dB} / \mathrm{km}$ & Fiber loss coefficient \\
\hline$L_{M u x}$ & $3 \mathrm{~dB}$ & Multiplexer loss \\
\hline$L_{\text {Demux }}$ & $3 \mathrm{~dB}$ & Demultiplexer loss \\
\hline$L_{\text {Switch }}$ & $3 \mathrm{~dB}$ & Optical switch loss \\
\hline$W_{M I N}$ & 4 & $\begin{array}{c}\text { Minimum number of wavelengths } \\
\text { per link }\end{array}$ \\
\hline$W_{M A X}$ & 40 & $\begin{array}{c}\text { Maximum number of wavelengths } \\
\text { per link }\end{array}$ \\
\hline
\end{tabular}

\section{A. Impact of Boolean operators in the networks with 14 nodes}

For the NFSNet case, we performed ten executions with 1000 generations each. Fig. 3 and Fig. 4 show the average value of the hypervolume for the Pareto Front obtained from NSGAII in for every combination of operators. We adopted Uniform Crossover 0.25 nomenclature for Uniform Crossover when crossover probability is $\mathrm{PC}=0.25$. The proposed operators will be called OR Crossover, XOR Crossover and $\sim$ XOR Crossover, and all possible combinations made between operators, such as OR and XOR Crossover, for example.

All crossover operators were assessed in two situations: with the mutation in the evolutionary process and without the mutation. These variations were performed to evaluate the impact of the mutation in the outcome of crossover operators. 


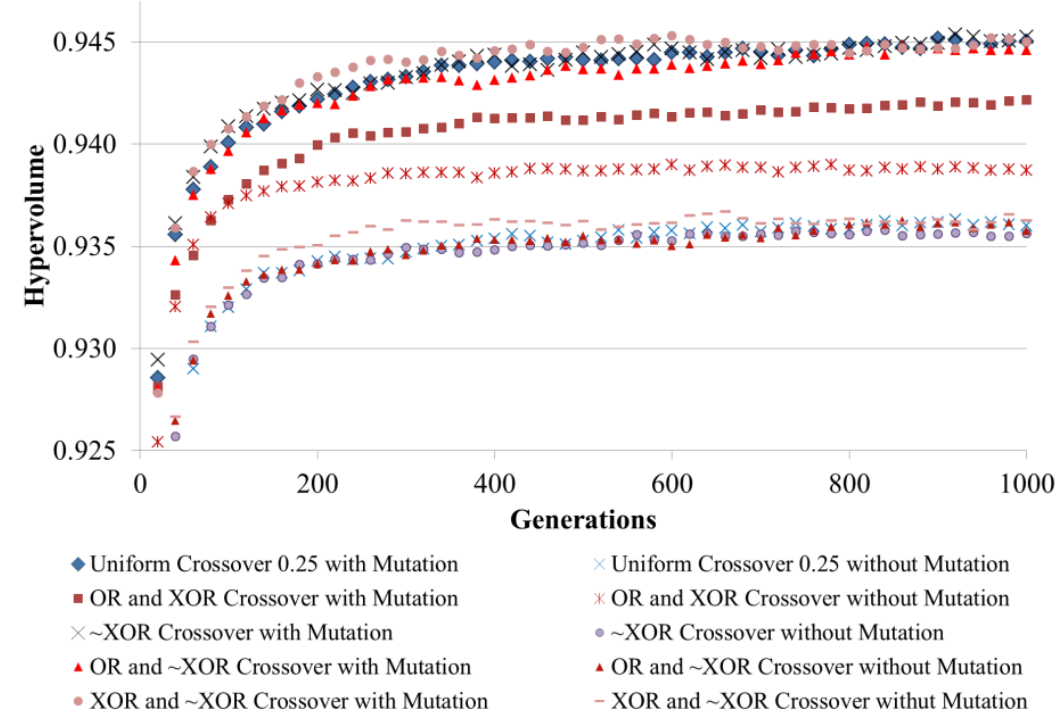

Fig. 3. Average value of the Hypervolume for different combinations of genetic operators for 14-node networks.

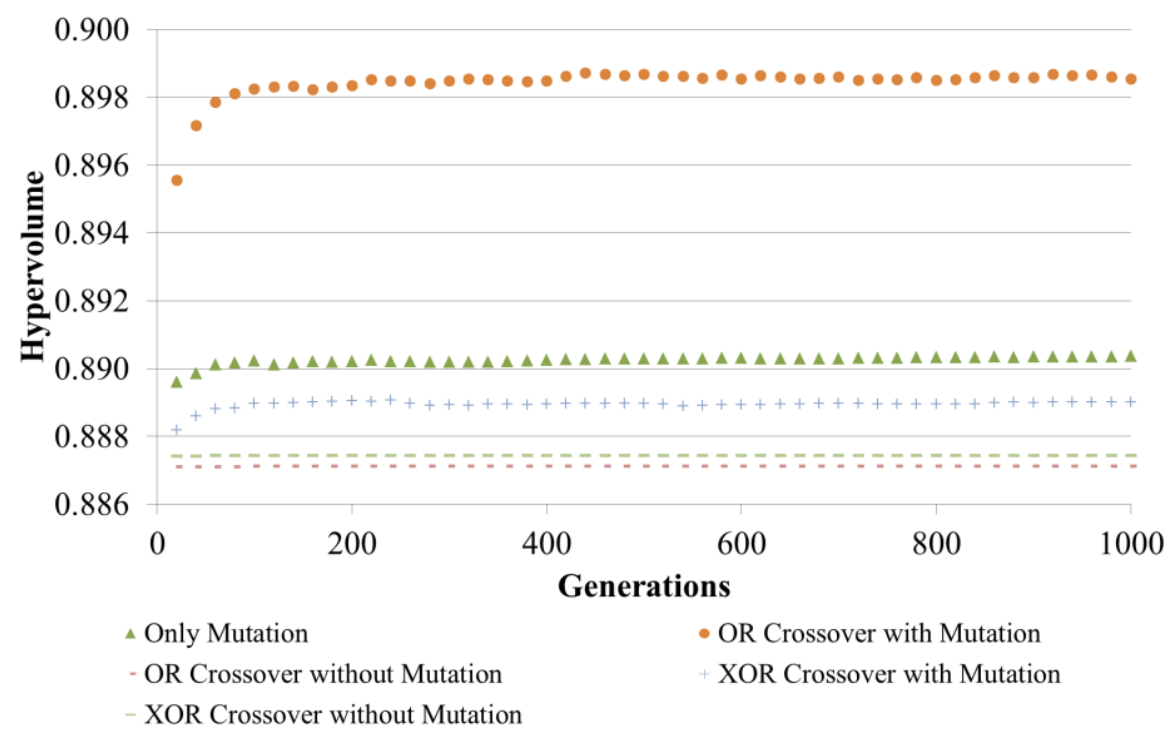

Fig. 4. Average value of the Hypervolume for OR Crossover, XOR Crossover and Mutation for 14-node networks.

In Fig. 3 we present the results for different combinations of genetic operators. Our goal is to analyze the impact of the mutation operator in our problem and to evaluate the benefit of using the crossover operator based on Boolean logics. For the cases related to the use of mutation the "XOR and $~ X O R$ Crossover" shows better results than the "Uniform Crossover 0.25".

When it is used the bitwise operations OR and XOR individually, we can observe that the best results are related to configuration "OR Crossover with Mutation". This analysis is summarized in the Fig. 4. This study is especially useful to emphasize that our new proposal is promising since the crossover based on Boolean logics acts really as a diversity operator and the curve from the "Mutation Only" is lower than the curve obtained by our proposal. 


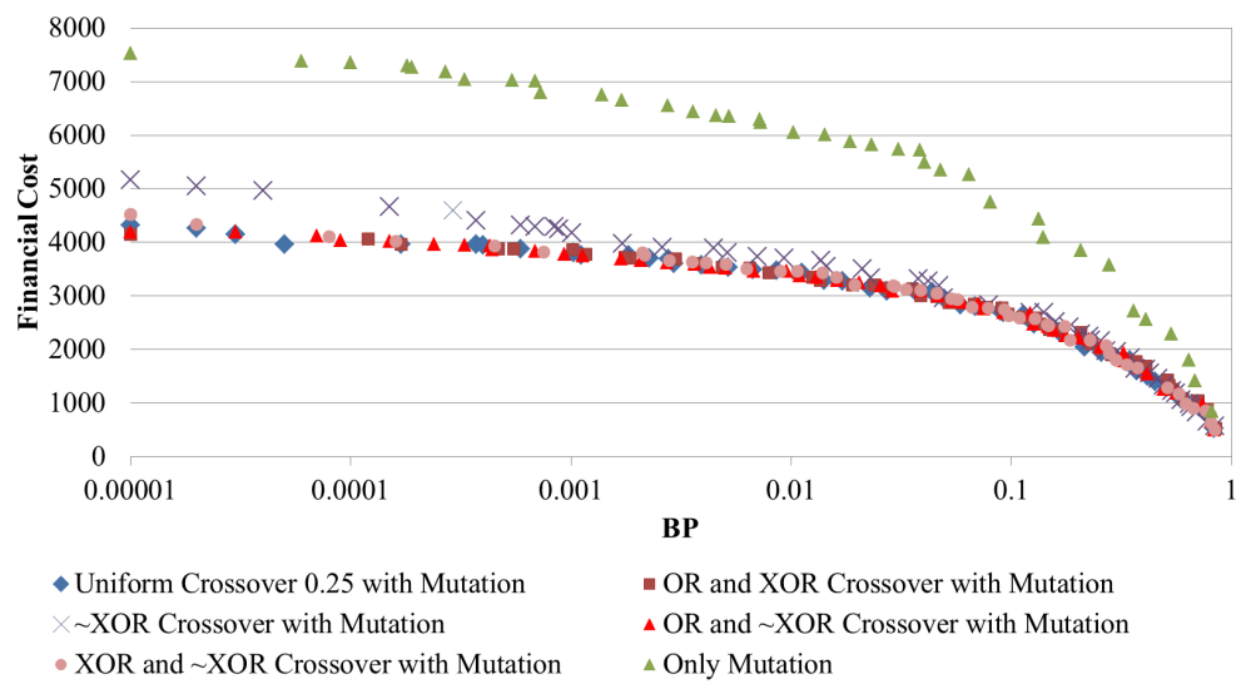

Fig. 5. Pareto Front for Uniform Crossover 0.25, OR/XOR Crossover, XOR Crossover, OR/ XOR Crossover and $\mathrm{XOR} / \mathrm{XOR}$ Crossover.

Fig. 5 shows the Pareto Fronts achieved by different combinations of genetic operators. One can observe that there is a big difference when it is used only the mutation to obtain the diversity of solutions. However, the slight difference between the Uniform Crossover and the crossover based on Boolean logics (shown in Fig. 3) is less perceptible when the objective space is inspected.

The main advantage of our proposal is related to the execution time of entire process. The summary of the analysis regarding execution time is shown in Table II. One can observe that all the crossover operators present similar execution times. However, the SIMTON simulator spends different execution time when it is applied to evaluate the fitness value for the extreme solutions of the Pareto Front regarding lower versus higher performance solutions. For example, solutions that present BP near to 1.0 are assessed using only 1000 request calls in the SIMTON. On the other hand, solutions that present BP near to $10^{-4}$ need $10^{7}$ request calls in the SIMTON to be assessed. Thus, when the genetic operator offers poor topologies for the new generations, there are different fitness evaluation times and the total execution time of the evolutionary algorithm is affected. The time spent on crossover operations are much lower than the total time, because the SIMTON spend some time more if the solutions obtained are not satisfactory. For example in the case of without mutation operations, could think of a shorter time spent when performing an operation less. However, when removing the mutation process also decreases the diversity of results, and therefore there is a worsening in the quality of results and some cases not meeting the restrictions BP, for example, then the simulator will work more time discarding several unsatisfactory cases due to poor quality solutions.

\section{B. Impact of Boolean operators in the networks with 34 nodes}

We performed a similar analysis for both the 34-node network and the 14-node. We depict in Fig. 6 
the results regarding of hypervolume for the most relevant combinations of genetic operators. We can observe that there is no perceptible difference between the executions related to different crossover operators. The most relevant difference is related to the absence of the crossover step in the evolutionary process (the case "Only Mutation" plotted as green triangles). Fig. 7 shows an approximation of the curves of crossover operators. It is possible to observe how close they are to each other.

Table II. Time spent in the simulations of networks of 14 nodes.

\begin{tabular}{c|c|c|c}
\hline Crossover Operators & $\begin{array}{c}\text { Total Time } \\
\text { (minutes) }\end{array}$ & $\begin{array}{c}\text { Total Time to Crossover } \\
\text { (minutes) }\end{array}$ & $\begin{array}{c}\text { Total Time to } \\
\text { Mutate } \\
\text { (minutes) }\end{array}$ \\
\hline Uniform 0.25 with Mutation & 9851 & 0.4460 & 0.6156 \\
\hline Uniform 0.25 Without Mutation & 13168 & 0.3633 & 0 \\
\hline$\sim$ XOR with Mutation & 4800 & 0.4559 & 0.4142 \\
\hline$\sim$ XOR without Mutation & 9831 & 0.3785 & 0 \\
\hline OR and XOR with Mutation & 9084 & 0.4332 & 0.6752 \\
\hline OR and XOR without Mutation & 11840 & 0.4019 & 0 \\
\hline OR and XOR with Mutation & 6252 & 0.4022 & 0.6069 \\
\hline OR and $\sim$ XOR without Mutation & 9739 & 0.3240 & 0.6196 \\
\hline XOR and XOR with Mutation & 6002 & 0.3949 & 0 \\
\hline XOR and $\sim$ XOR without Mutation & 9606 & 0.3361 & \\
\hline
\end{tabular}

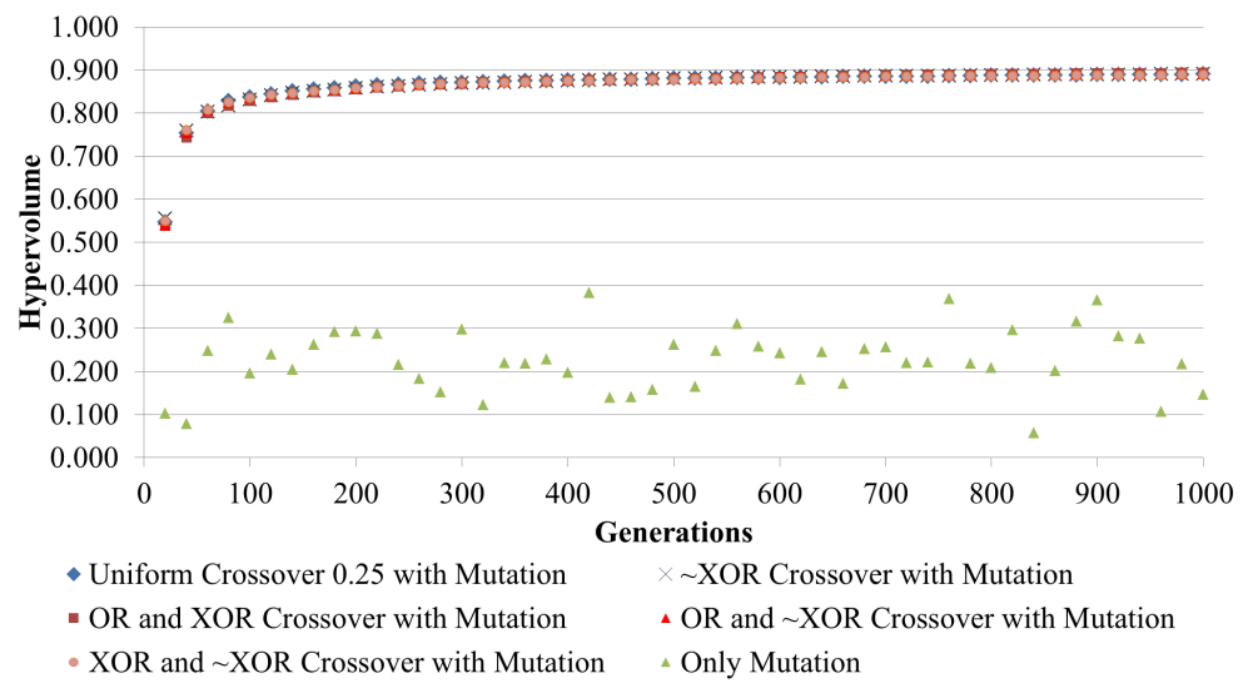

Fig. 6. Average value of the Hypervolume for different combinations of genetic operators for 34-node networks.

In Fig. 8 we present the Pareto Fronts for the same configuration of genetic operators. One can observe that the assessed crossover operators presented similar trade-offs regarding cost and blocking probability. 


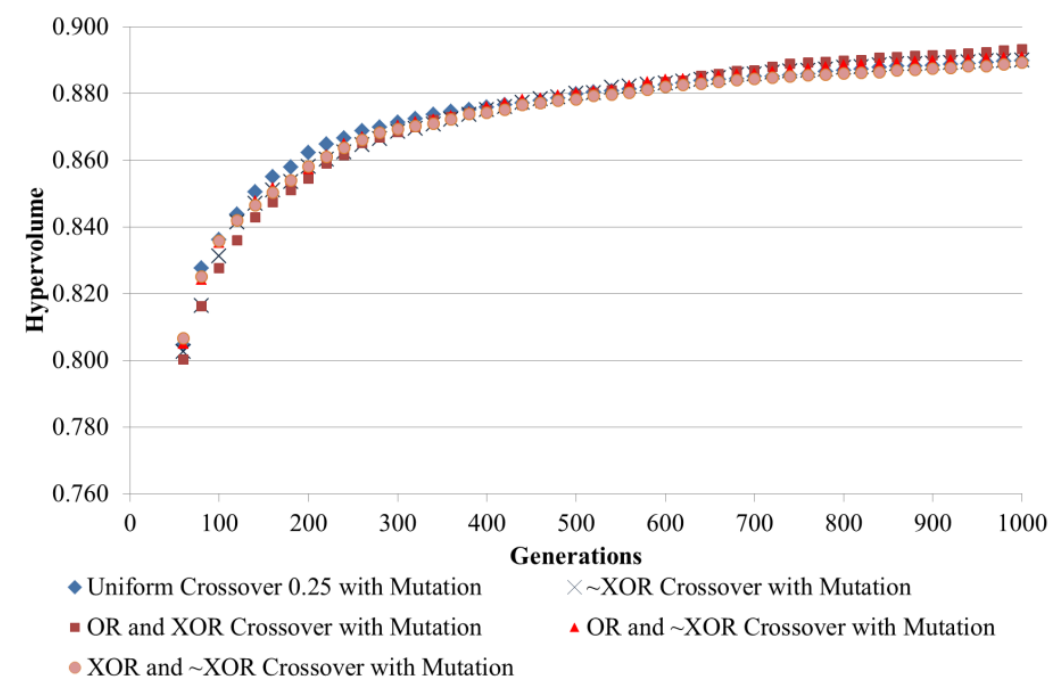

Fig. 7. Approximate graph to average value of the Hypervolume for different combinations of genetic operators for 34-node networks, excluding the mutation only operator to provide a zoom in the region of interest.

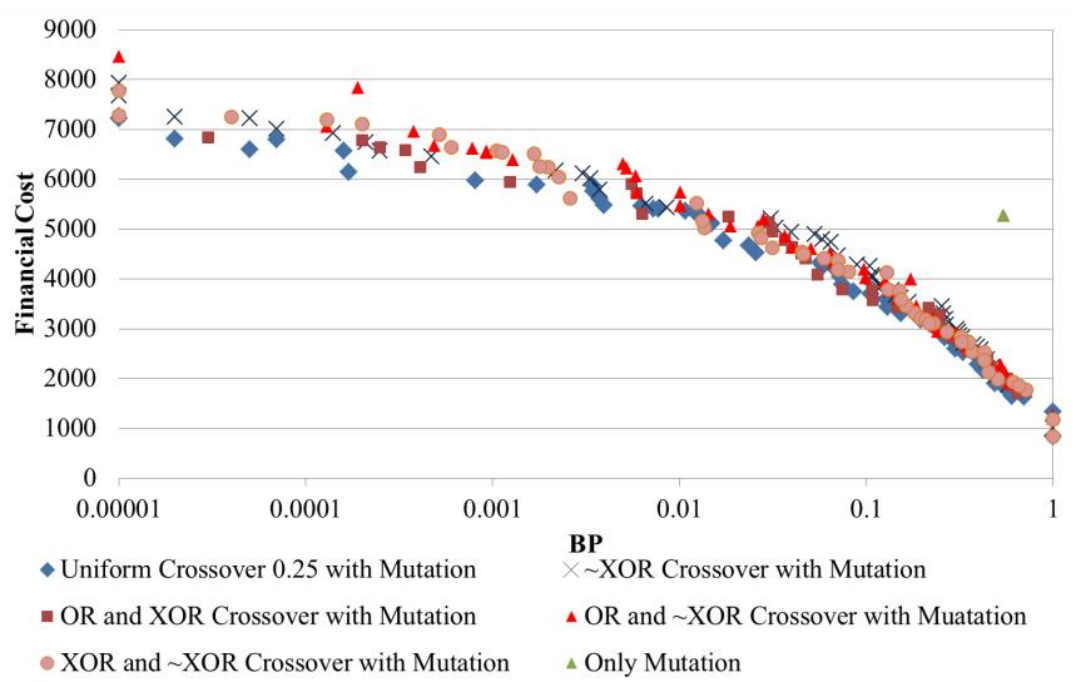

Fig. 8. Pareto Front for Uniform Crossover 0.25, OR/XOR Crossover, XOR Crossover, OR/ XOR Crossover, and XOR/ XOR Crossover.

Table III shows the execution time for the design of the 34-node network. We can observe that the execution time is very similar for the "Uniform Crossover" and "OR and XOR Crossover", but in the cases where combinations of Boolean operator are deployed, the execution times are reduced. In this case, the crossover based on Boolean logics is an alternative to the traditional approaches.

The execution time needed for the crossover and mutation operations are much lower then the time to evaluate a solution in the SIMTON simulator. One can think that the no use of the mutation operator would lead to a smaller execution time. However, without mutation, we have a decrease in the diversity of the meta-heuristic population, and as a consequence, more offsprings do not meet the Brazilian Microwave and Optoelectronics Society-SBMO received 08 May 2016; for review 12 May 2016 ; accepted 06 Sept 2016 Brazilian Society of Electromagnetism-SBMag 
$\mathrm{BP}$ requirements. In these cases, the simulator will work harder to discard non-satisfatory low-quality solutions.

TABLE III. TIME SPENT IN THE SIMULATIONS OF NETWORKS OF 34 NODES.

\begin{tabular}{c|c|c|c}
\hline Crossover operators & $\begin{array}{c}\text { Total time } \\
\text { (minutes) }\end{array}$ & $\begin{array}{c}\text { Total time to } \\
\text { crossover } \\
\text { (minutes) }\end{array}$ & $\begin{array}{c}\text { Total time to } \\
\text { Mutate } \\
\text { (minutes) }\end{array}$ \\
\hline Uniform 0.25 with Mutation & 15561 & 0.0664 & 0.1191 \\
\hline$\sim$ XOR with Mutation & 9740 & 0.0593 & 0.09771 \\
\hline OR and XOR with Mutation & 14104 & 0.0666 & 0.1200 \\
\hline OR and $\sim$ XOR with Mutation & 7237 & 0.05646 & 0.0998 \\
\hline XOR and $\sim$ XOR with Mutation & 8900 & 0.0446 & 0.0841 \\
\hline
\end{tabular}

\section{CONCLUSIONS}

In this paper, we analyzed the impact of a new crossover operator based on Boolean logics that can be applied to optimize problems related to graphs in evolutionary algorithms. We evaluated the proposal to design optical networks with 14 and 34 nodes. The OR and XOR operator shown similar results when it is compared with the Uniform Crossover, but the whole execution time was lower in some scenarios related to the 14-node case. The cases using ${ }^{\sim}$ XOR shown better results and the whole execution time was much lower than Uniform Crossover. When we take into account that this type of crossover operator has never been studied before, we consider this study as the first investigation towards an alternative to optimize problems related to graphs in meta-heuristics. The proposed topological operators may be useful for designing networks in which there is installed equipment and expansion of existing networks, which may use the structure and apply this approach to find new paths to the data flow, bringing benefits to all networks.

The purpose of this study was to evaluate only Boolean operators OR, XOR and $\sim$ XOR, but a future work would test other Boolean operators and evaluate their behavior.

Adjustments can be made in the design algorithm of optical networks for the intersection can be done in some regions of interest of the graph and not the network as a whole. Based on the same idea to perform a combination of parts of the graph with the crossing OR and XOR operators, it might also be tested application directly to network nodes, i.e., check the desired bonds present in a specific node, and perform the combination according to the desired design while maintaining important connections or eliminating unnecessary connections.

The topological operators proposed in this paper can be useful for the design or expansion of realworld networks. In general, the topological structure of the network defines the bottlenecks for the flow of information within the network. Therefore, more adapted operators can lead to better choices for finding such topologies. 


\section{ACKNOWLEDGMENT}

The authors acknowledge the financial support from CNPq, CAPES, UFPE, UFRPE and UPE.

\section{REFERENCES}

[1] R. Ramaswami e K. N. Sivarajan, Optical Networks: A Practical Perspective., 3rd edition ed., Morgan Kaufmann, 2010.

[2] D. R. B. Araújo, C. J. A. Bastos-Filho e J. F. Martins-Filho, "An Evolutionary Approach with Surrogate Models and Network Science Concepts to Design Optical Networks," em Engineering Application of Artificial Intelligence, 2015.

[3] D. R. B. Araújo, J. F. Martins-Filho e C. J. A. Bastos-Filho, "New Graph Model to Design Optical Networks," Communications Letters, IEEE, pp. 19(12): 2130-2133, 2015.

[4] H. Liu e F. A. Tobagi, "Physical Topology Design for All-Optical networks," Optical Switching and Networking, pp. 5(4): 219-231, 2008.

[5] R. M. Morais, C. Pavan, A. N. Pinto e C. Raquejo, "Genetic Algorithm for the Topological Design of Survivable Optical Transport Networks.," Journal of Optical Communications and Networking, pp. 3(1):17-26, 2011.

[6] D. R. B. Araújo, C. J. A. Bastos-Filho, E. A. Barboza, D. A. R. Chaves e J. F. Martins-Filho, "An Efficient Multiobjective Evolutionary Optimizer to Design All-Optical Networks Considering Physical Impairmens and CAPEX," em Intelligent System Design and Applications (ISDA), 11th International Conference, 2011.

[7] M. Gerla e L. Kleinrock, "On the Topological Design of Distributed Computer Networks," Communications, IEEE Transactions, pp. 25(1):48-60, 1977.

[8] R. R. Boorstyn e H. Frank, "Large-Scale Network Topological Optimization," Communcations, IEEE Transactions, pp. 25(1):29-47, 1977.

[9] D. R. B. Araújo, C. J. A. Bastos-Filho, E. A. Barboza, D. A. R. Chaves e J. F. Martins-Filho, "A Performance Comparison of Multi-Objective Computacional Intelligence in Multicriteria Decision-MAking (MDCM)," em IEEE Simposyum, 2011.

[10] K. Man, K. Tang e S. Kwong, "Genetic Algorithms: Concepts and Applications (in Engineering Design),” em IEEE Transactions on Industrial Eletronics, 1996.

[11] N. J. S. Lima, D. R. B. Araújo, J. F. Martins-Filho e C. J. A. Bastos-Filho, "Towards Using Boolean Operators on Graphs to Generate Network Topologies," em SBMO/IEEE MTT-S International Microwave an Optoeletronics Conference (IMOC), Porto de Galinhas, Ipojuca-PE, 2015.

[12] T. G. Lewis, Network Science - Theory and Applications, John Wiley \& Sons, 2009.

[13] J. Knowles e D. Corne, "On Metrics for Comparing Non-Dominated Sets," em Congress on Evolutionary Computation (CEC 2002), 2002.

[14] E. Zitler, L. Thiele, M. Laumanns, C. M. Fonseca e V. G. da Fonseca, "Performance Assessment of Multiobjetive Optimizaers: An Analysis and Review.," IEEE Transactions on Evolutionary Computation, pp. 7(2): 117-132, 2003.

[15] E. Zitler, "Evolutionary Algorithms for Multiobjective Optimization: Methods and Applications.," Switzerland, 1999.

[16] K. Deb, A. Pratap, S. Agarwal e T. Meyarivan, “A Fast and Elitist Multiobjective Genetic Algorithm: NSGAII," em Evolutionary Computation, IEEE Transactions, 2002.

[17] C. A. Coello-Coello, G. B. Lamont e D. A. V. Veldhuizen, Evolutionary Algorithms for Solving Multi-Objective Problems, $2^{a}$ ed., Springer, 2007.

[18] K. Deb e H. Jain, "An Evolutionary Many-Objective Optimization Algorithm Using Reference-Point-Based NonDominated Sorting Approach, part i: Solving Problems with Box Constraints," em Evolutionary Compuation, IEEE Transactions, 2014.

[19] D. A. R. Chaves, "Projeto de Redes Ópticas de Alta Capacidade Utilizando Técnicas de Otimização Bioinspiradas," Recife, 2012.

[20] D. R. B. Araújo, "Comparação de Algoritmos de Otimização Multi-Objetivos Aplicados a Projetos de Redes Ópticas,” Recife-PE, 2011.

[21] D. A. R. Chaves, C. J. A. Bastos-Filho e J. F. Martins-Filho, "Multiobjective Physical Topology Design of All-Optical Networks Considering QoS and CAPEX.," em Optical Fiber Communication (OFC), 2010 Conference on (OFC/NFOEC), 2010. 